\title{
Calpain-Calcineurin-Nuclear Factor Signaling and the Development of Atrial Fibrillation in Patients with Valvular Heart Disease and Diabetes
}

\author{
Yong Zhao, ${ }_{1}^{1}$ Guo-ming Cui, ${ }^{2}$ Nan-nan Zhou, ${ }^{1}$ Cong Li, ${ }^{3}$ Qing Zhang, ${ }^{1}$ Hui Sun, ${ }^{4}$ Bo Han, \\ Cheng-wei Zou, ${ }^{6}$ Li-juan Wang, ${ }^{1}$ Xiao-dong Li, ${ }^{1}$ and Jian-chun Wang ${ }^{1}$ \\ ${ }^{1}$ Department of Geriatric Cardiology, Shandong Provincial Hospital Affiliated to Shandong University, Jinan 250021, China \\ ${ }^{2}$ Shandong Medicinal Imaging Research Institute, Jinan 250021, China \\ ${ }^{3}$ Intensive Care Unit, Shouguang People's Hospital, Weifang 262700, China \\ ${ }^{4}$ Department of Cardiology, Jinan Central Hospital Affiliated to Shandong University, Jinan, Shandong 250013, China \\ ${ }^{5}$ Department of Cardiac Surgery, The 4th Hospital of Jinan, Jinan 250031, China \\ ${ }^{6}$ Department of Cardiac Surgery, Shandong Provincial Hospital Affiliated to Shandong University, Jinan 250021, China
}

Correspondence should be addressed to Jian-chun Wang; wangjianchun@medmail.com.cn

Received 17 December 2015; Accepted 17 March 2016

Academic Editor: Dake Qi

Copyright (C) 2016 Yong Zhao et al. This is an open access article distributed under the Creative Commons Attribution License, which permits unrestricted use, distribution, and reproduction in any medium, provided the original work is properly cited.

\begin{abstract}
Calpain, calcineurin (CaN), and nuclear factor of activated T cell (NFAT) play a key role in the development of atrial fibrillation. Patients with valvular heart disease (VHD) are prone to develop atrial fibrillation (AF). Thus, our current study was aimed at investigating whether activation of calpain-CaN-NFAT pathway is associated with the incidence of AF in the patients with VHD and diabetes. The expressions of calpain 2 and alpha- and beta-isoforms of CaN catalytic subunit (CnA) as well as NFAT-c3 and NFAT-c4 were quantified by quantitative reverse transcription-polymerase chain reaction in atrial tissues from 77 hospitalized patients with VHD and diabetes. The relevant protein content was measured by Western blot and calpain 2 in human atrium was localized by immunohistochemistry. We found that the expressions of calpain 2, CnA alpha and CnA beta, and NFAT-c3 but not NFAT-c4 were significantly elevated in the samples from patients with AF compared to those with sinus rhythm (SR). Elevated protein levels of calpain 2 and $\mathrm{CnA}$ were observed in patients with $\mathrm{AF}$, and so was the enhanced localization of calpain 2 . We thereby concluded that $\mathrm{CaN}$ together with its upstream molecule, calpain 2, and its downstream effector, NFAT-c3, might contribute to the development of AF in patients with VHD and diabetes.
\end{abstract}

\section{Introduction}

Atrial fibrillation (AF) is the most common abnormal heart rhythm in human and imposes a substantial burden on population health. The odds of developing a stroke are almost 5 -fold higher in patients with AF than in those free of AF [1]. However, the underlying pathophysiological mechanisms of $\mathrm{AF}$ are not entirely understood. It may be associated with ion channel dysfunction, $\mathrm{Ca}^{2+}$-handling abnormalities, structural remodeling, and autonomic neural dysregulation [2].

Calpains represent a family of intracellular $\mathrm{Ca}^{2+}$-activated cysteine proteases and the 15 family members have been proposed to be actively involved in the occurrence of $\mathrm{AF}$ [3]. Calcineurin $(\mathrm{CaN})$ is a $\mathrm{Ca}^{2+} /$ calmodulin activated serine/threonine protein phosphatase and can be activated by endogenous calpain in human heart [4]. It is composed of a $\approx 60$ - $\mathrm{kDa}$ catalytic A subunit $(\mathrm{CnA})$ and a $19 \mathrm{kDa}$ regulatory $B$ subunit $(\mathrm{CnB})$. Three isoforms have been identified in $\mathrm{CnA}(\alpha, \beta$, and $\gamma)$ and 2 isoforms in $\mathrm{CnB}(\mathrm{CnB} 1$ and $\mathrm{CnB2}$ ) [5]. CnA has been reported to modulate transcription through dephosphorylating and facilitating translocation of nuclear factor of activated T cells (NFATs). Previous studies also indicated that $\mathrm{CaN}$ activity was increased following NFAT-c3 and NFAT-c4 translocation into the nucleus in a porcine model of AF, suggesting that the activation of 
CaN-NFAT signal transduction pathway might be involved in the pathogenesis of AF [6].

Many pathological processes contribute to the development of AF. For example, valvular heart disease (VHD) has been identified to be a risk factor of AF [7]. In addition, a prospective cohort study suggested that diabetes also significantly increased the incidence of AF by $35 \%$ [8]. Thus, in the current study, we aimed to investigate the relationship between calpain-CaN-NFAT pathway and the development of AF in patients with VHD and diabetes. We evaluated the expressions of calpain, $\mathrm{CaN}$, and NFATs in atrial tissues from patients with and without chronic persistent AF.

\section{Methods}

2.1. Patients and Atrial Samples. This human study was conducted in accordance with the principles outlined in Declaration of Helsinki. The research protocol was approved by the Ethics Committee of Provincial Hospital Affiliated to Shandong University. Written informed consents for research use of discarded atrial tissues were provided by all the patients or their guardians. Within 2007-2013, left atrial appendages were dissected from 77 patients with VHD and diabetes during the mitral/aortic valve replacement surgeries. All these patients had their cardiac functions in New York Heart Association (NYHA) II-III function class and none of them ever received class I or III antiarrhythmic drugs. These atrial samples were subsequently frozen in liquid nitrogen and stored at $-80^{\circ} \mathrm{C}$ for a further analysis.

Demographic and clinical data were retrieved by a chart review. Blood pressure (BP) was measured with a mercury sphygmomanometer on the right arm of each patient resting in a supine posture. And the heart rate was documented. Prior to the surgeries, overnight fasting blood samples were collected to measure hemoglobin $(\mathrm{Hb})$, alanine transferase (ALT), creatinine ( $\mathrm{Cr}$ ), low-density lipoprotein cholesterol (LDL-C), fast blood glucose (FBG), international normalized ratio (INR), angiotensin I and angiotensin II (Ang I and Ang II), aldosterone, and brain natriuretic peptide (BNP). Atrial fibrillation was confirmed with electrocardiography. Transthoracic echocardiography through Philips Sonos 5500 Ultrasound System (HP Agilent, SC, USA) was operated by an experienced sonographer to assess the diastolic diameter of each heart chamber, the thickness of interventricular septum (IVS) and left ventricular posterior wall (LVPW), the status of valves, and the left ventricular ejection fraction (LVEF).

\subsection{Gene Expression Measurement by Real-Time Quantitative} Reverse Transcription-Polymerase Chain Reaction ( $q P C R)$. Frozen left atrial appendage was thawed and removed from RNAIater stabilization reagent. About $50 \mathrm{mg}$ of specimens was minced on ice and homogenized in $1 \mathrm{~mL}$ TRIzol (TaKaRa Bio, Tokyo, Japan). After addition of $200 \mu \mathrm{L}$ chloroform, the mixture was incubated at room temperature for $3 \mathrm{~min}$ and centrifuged at $12,000 \mathrm{~g}$ for $15 \mathrm{~min}$ at $4^{\circ} \mathrm{C}$. Upper aqueous phase $(400 \mu \mathrm{L})$ was transferred to a $1.5 \mathrm{~mL}$ RNase-free tube and mixed with 1 volume of isopropanol. The homogenate sat on ice for $10 \mathrm{~min}$ and was centrifuged at $12,000 \mathrm{~g}$ for $10 \mathrm{~min}$ at $4^{\circ} \mathrm{C}$. Total RNA was precipitated and then washed by $1 \mathrm{~mL}$ $75 \%$ ethanol. After centrifugation at 7,500 g for $5 \mathrm{~min}$ at $4^{\circ} \mathrm{C}$ and addition of ethanol were repeated twice, the recovered RNA pellet was dissolved in diethylpyrocarbonate- (DEPC-) treated sterile water. Total RNA was quantified spectrophotometrically at $260 \mathrm{~nm}$ and its quality was validated by the $\mathrm{OD}_{260} / \mathrm{OD}_{280}$ absorption ratio in the range of 1.8 to 2.0.

RNAs were reverse-transcribed into cDNA using PrimeScript $^{\mathrm{TM}}$ RT Master Mix (TaKaRa Bio, Tokyo, Japan). During the synthesis of cDNA, a mix of $520 \mu \mathrm{g}$ RNA, $2 \mu \mathrm{L} 5 \times \mathrm{g}$ DNA Eraser Buffer, and $1 \mu \mathrm{L}$ gDNA Eraser was incubated in a final volume of $10 \mu \mathrm{L}$ with RNase free $\mathrm{ddH}_{2} \mathrm{O}$ at $42^{\circ} \mathrm{C}$ for $4 \mathrm{~min}$ to minimize the contamination of genomic DNA. Then $1 \mu \mathrm{L}$ PrimeScript RT Enzyme Mix I, $1 \mu \mathrm{L}$ RT Primer Mix, $4 \mu \mathrm{L} 5 \mathrm{x}$ PrimeScript Buffer, and $4 \mu \mathrm{L}$ RNase free $\mathrm{ddH}_{2} \mathrm{O}$ were added for 15 -minute incubation at $37^{\circ} \mathrm{C}$, followed by inactivation of reverse transcriptase at $85^{\circ} \mathrm{C}$ for $5 \mathrm{sec}$, and stored at $4^{\circ} \mathrm{C}$.

Real-time quantitative PCR was performed using a LightCycler $^{\circledR} 480$ Real-Time PCR System (Roche Diagnostics, $\mathrm{GmbH}$, Penzberg, Germany). Briefly, the reaction mixtures $(20 \mu \mathrm{L})$ included $2 \mu \mathrm{L}$ cDNA, $10 \mu \mathrm{L} 2 \mathrm{x}$ SYBR $^{\circledR}$ Premix Ex $\mathrm{Taq}^{\mathrm{TM}}(\mathrm{TaKaRa}$ Bio, Tokyo, Japan), and $0.4 \mu \mathrm{L} 10 \mu \mathrm{mol} / \mathrm{L}$ specific sense and antisense primers listed in Table 1. Cycle conditions were predenaturation at $95^{\circ} \mathrm{C}$ for $30 \mathrm{sec}$, then 40 cycles of denaturation at $94^{\circ} \mathrm{C}$ for $5 \mathrm{sec}$, annealing at $60^{\circ} \mathrm{C}$ for $20 \mathrm{sec}$, and extension at $95^{\circ} \mathrm{C}$ for $5 \mathrm{sec}$, followed by a final extension at $60^{\circ} \mathrm{C}$ for $1 \mathrm{~min}$. The specificity of PCR product was examined by $1.5 \%$ agarose gel electrophoresis and melting curve analysis. Each assay was analyzed in triplicate, and the relative expression levels were calculated by the $2^{-\Delta \Delta C t}$ method using $\beta$-actin as endogenous control for normalization of cDNA contents.

2.3. Protein Quantification by Western Blot. About $50 \mathrm{mg}$ frozen samples from left atrial appendage were grinded in liquid nitrogen and subsequently homogenized in $1.5 \mathrm{~mL}$ lysis buffer (50 mM Tris- $\mathrm{HCl}, 100 \mathrm{mM} \mathrm{NaCl}, 1 \%$ NP-40, 0.1\% SDS, $1 \mathrm{mM}$ phenylmethylsulfonyl fluoride, $\mathrm{pH}$ 7.4). Homogenates were incubated for $30 \mathrm{~min}$ on ice and centrifuged at 15,000 $\mathrm{g}$ for $30 \mathrm{~min}$ at $4^{\circ} \mathrm{C}$. The protein content from supernatants was determined using bicinchoninic acid (BCA) method. After being denatured at $100^{\circ} \mathrm{C}$ for $10 \mathrm{~min}$, extracted proteins ( $40 \mu \mathrm{g}$ per lane) were separated by $8 \%-10 \%$ gradient sodium dodecyl sulfate-polyacrylamide gel electrophoresis (SDSPAGE) at $100 \mathrm{~V}$ for $1.5 \mathrm{~h}$ and transferred onto polyvinylidene fluoride (PVEF) membranes (Millipore, Massachusetts, USA) at $4^{\circ} \mathrm{C}$ and $200 \mathrm{~mA}$. Membranes were blocked in $5 \%$ w/v nonfat dry milk TBST buffer (Tris-base $1.21 \mathrm{~g} / \mathrm{L}, \mathrm{NaCl}$ $8.78 \mathrm{~g} / \mathrm{L}, 1 \mathrm{~mL}$ Tween-20/L, $\mathrm{pH} 7.6$ ) at room temperature for $1 \mathrm{~h}$ and incubated for overnight at $4^{\circ} \mathrm{C}$ with primary antibodies: mouse anti-human $\mathrm{CaN}$ monoclonal antibody (BD Transduction Laboratories, San Diego, CA, USA; $1: 250$ ), rabbit anti-human calpain 2 polyclonal antibody (Abgent, San Diego, CA, USA; $1: 1000$ ), and mouse anti-human $\beta$-actin monoclonal antibody (Zhongshan Jinqiao Bio-Technology Co. Ltd., Beijing, China; $1: 1000)$. In the next day, membranes were washed with TBST buffer 3 times and incubated with horseradish peroxidase-conjugated goat anti-rabbit/mouse secondary antibody (Zhongshan Jinqiao Bio-Technology Co. 
TABLE 1: Primer sequences were used in qPCR experiments.

\begin{tabular}{lcl}
\hline Gene & Product size $(\mathrm{bp})$ & Primer sequence \\
\hline Calpain 2 & 165 & $\begin{array}{l}\text { F: } 5^{\prime} \text {-CTGGGGCTGAAGGAGTTCTAC } \\
\text { R: } 5^{\prime} \text {-GATGACTTGGTGGAGTTGACAG }\end{array}$ \\
\hline CnA $\alpha$ isoform & 112 & $\begin{array}{l}\text { F: } 5^{\prime} \text {-CAGGAACATTTCACTCACAACACAG } \\
\text { R: } 5^{\prime} \text {-CGTGGGCTCGGAGTATAGATAACA }\end{array}$ \\
\hline CnA $\beta$ isoform & \multirow{2}{*}{119} & $\begin{array}{l}\text { F: } 5^{\prime} \text {-TGGATGTCTTCACGTGGTCTTTAC } \\
\text { R: } 5^{\prime} \text {-ATCAAACTGGTCTTCACCTTCAGTC }\end{array}$ \\
\hline NFAT-c3 & \multirow{2}{*}{137} & $\begin{array}{l}\text { F: } 5^{\prime} \text {-CATCGAGCCCATTATGAAACTGAA } \\
\text { R: } 5^{\prime} \text {-CGATCATCTGCTGTCCCAATAAAC }\end{array}$ \\
\hline NFAT-c4 & \multirow{2}{*}{$\begin{array}{l}\text { F: } 5^{\prime} \text {-AGCCTGACACACCGTAGGTACTGA } \\
\text { R:-actin }\end{array}$} & $\begin{array}{l}\text { F: }: 5^{\prime} \text {-ACACTGCAAATGCCCCGGATCTACGAGGGG } \\
\text { R: } 5^{\prime} \text {-ATGAGTGAGTTGAAGGTAGTTTCGTGGAT }\end{array}$ \\
\hline
\end{tabular}

$\mathrm{CnA}$, subunit A of calcineurin; F, forward primer; NFAT, nuclear factor of activated T cells; R, reverse primer.

Ltd., Beijing, China; $1: 1000)$ at room temperature for $1 \mathrm{~h}$. After another 3 washes with TBST buffer, signals were detected with enhanced chemiluminescence (ECL) HRP substrate (Millipore, Boston, MA, USA) and quantified by densitometry with Fujifilm Las-4000 Luminescent Image Analyzer (Fuji, Tokyo, Japan).

2.4. Immunohistochemical Localization of Calpain 2. Dissected tissues were fixed in 10\% paraformaldehyde and made into $20 \mu \mathrm{m}$ thick paraffin-embedded sections. After being heated for $30 \mathrm{~min}$ at $65^{\circ} \mathrm{C}$, slides were deparaffinized with turpentine oil, rehydrated with gradient ethanols, rinsed with distilled water 3 times for 5 min each, washed with phosphate buffered saline (PBS) for $5 \mathrm{~min}$, and immersed into preheated retrieval solution $\left(98^{\circ} \mathrm{C}, \mathrm{pH}=6.0\right)$ for $15 \mathrm{~min}$. Upon cooling to room temperature, slides were rinsed gently with PBS 3 times for $5 \mathrm{~min}$ each and then incubated with $3 \% \mathrm{H}_{2} \mathrm{O}_{2}$ at $37^{\circ} \mathrm{C}$ for $30 \mathrm{~min}$ to quench endogenous peroxidase activity. Rinsed for another 3 times with PBS, the slides were added with normal goat serum (Zhongshan Jinqiao Bio-Technology Co. Ltd., Beijing, China) to block nonspecific antigens for $30 \mathrm{~min}$ at $37^{\circ} \mathrm{C}$. Excess blocking serum was wiped away and the slides were incubated with rabbit polyclonal primary antibodies to calpain 2 (Abgent, San Diego, CA, USA; 1:100) overnight at $4^{\circ} \mathrm{C}$. After incubation at $37^{\circ} \mathrm{C}$ for $30 \mathrm{~min}$ and 3 washes with PBS, biotinylated secondary antibodies (Zhongshan Jinqiao Bio-Technology Co. Ltd., Beijing, China) were added to incubate at $37^{\circ} \mathrm{C}$ for $30 \mathrm{~min}$ followed by another 3 washes with PBS. The tissue sections were immersed with 3,3'diaminobenzidine (DAB) Chromogen Solution (Zhongshan Jinqiao Bio-Technology Co. Ltd., Beijing, China) for 3 min and mounted with nuclear counterstain hematoxylin for 3 min. After being dehydrated with ethanol and cleared with turpentine oil, stained tissue was covered with a coverslip and visualized under a light microscope. The specificity of immunostaining was controlled by substituting the primary antibodies with PBS. Calpain 2-positive cells were identified by the presence of brown granules in the cytoplasm. Image-Pro Plus Version 6.0 image analysis system (Media Cybernetics, Inc., Silver Spring, MD, USA) was adopted to evaluate semiquantitatively the average optical density (OD) of the positive stained areas in 3 random visual fields (magnification, $\times 400$ ) from each histological section.

2.5. Statistical Analysis. Continuous variables were expressed as Mean \pm Standard Deviation (SD). SPSS 17.0 (SPSS Inc., Chicago, IL, USA) was used for data analysis. The difference between two groups was evaluated by unpaired Student's $t$ test and the comparison among multigroups was tested by one-way analysis of variance (ANOVA). Categorical variables were summarized as frequency and percentage and evaluated using Chi-square or Fisher's exact test. Pearson correlation coefficient $(r)$ was used to assess the association between parameters. $P<0.05$ was considered as significant difference.

\section{Results}

3.1. Clinical Characteristics of Patients with AF and SR. The patients include 42 males and 35 females of the age of $50.73 \pm$ 7.65 years. Forty-five of them were diagnosed with AF and 32 with sinus rhythm (SR). Tables 2 and 3 show the preoperative clinical and laboratory characteristics of these patients. The rest heart rates and levels of INR, BNP, and Ang I and Ang II were significantly higher in patients with AF compared to those with SR. These increased parameters were paralleled with exacerbated cardiac dysfunction characterized with augmented left and right atrium and attenuated left ventricular ejection fraction (LVEF).

3.2. The Gene Expressions of Calpain 2, CaN, and NFATs. At the transcription level, the expressions of calpain 2 (128.37 \pm $63.01 \%$ versus $100 \pm 36.07 \%, P=0.015), \mathrm{CnA} \alpha(154.90 \pm$ $100.38 \%$ versus $100 \pm 98.44 \%, P=0.020), \mathrm{CnA} \beta(159.16 \pm$ $144.34 \%$ versus $100 \pm 73.36 \%, P=0.037)$, and NFAT-c3 $(130.79 \pm 100.00 \%$ versus $100 \pm 85.48 \%, P=0.028)$ were significantly increased in patients with AF compared to those with SR. However, there was no statistical difference in the expression of NFAT-c4 between these two groups (119.40 \pm $85.51 \%$ versus $100 \pm 89.82 \%, P=0.340$; Figure 1$)$. These findings suggested that Calpain 2, $\mathrm{CnA} \beta$, and NFAT-c3 rather 
TABLE 2: Clinical assessments of patients.

\begin{tabular}{|c|c|c|c|}
\hline & $\mathrm{AF}(n=45)$ & $\mathrm{SR}(n=32)$ & $P$ \\
\hline Age (years) & $51.20 \pm 6.33$ & $50.06 \pm 9.26$ & 0.524 \\
\hline Sex (male, \%) & $24(53.33 \%)$ & $18(56.25 \%)$ & 0.800 \\
\hline Cigarette smoking & $9(20.00 \%)$ & $7(21.88 \%)$ & 0.842 \\
\hline Alcohol drinking & $13(28.89 \%)$ & $11(34.38 \%)$ & 0.609 \\
\hline \multicolumn{4}{|l|}{ Diseases } \\
\hline MS & $14(31.11 \%)$ & $10(31.25 \%)$ & \multirow{4}{*}{0.505} \\
\hline $\mathrm{MR}$ & $22(48.89 \%)$ & $18(56.25 \%)$ & \\
\hline $\mathrm{MS}+\mathrm{MR}$ & $6(13.33 \%)$ & $4(12.50 \%)$ & \\
\hline $\mathrm{MS}+\mathrm{MR}+\mathrm{AS} / \mathrm{AR}$ & $3(6.67 \%)$ & 0 & \\
\hline \multicolumn{4}{|l|}{ Physical examination } \\
\hline Systolic BP (mmHg) & $120.22 \pm 8.40$ & $123.31 \pm 12.62$ & 0.201 \\
\hline Diastolic BP (mmHg) & $75.98 \pm 8.01$ & $73.06 \pm 10.54$ & 0.172 \\
\hline Rest heart rates (bpm) & $81.13 \pm 14.74$ & $74.03 \pm 8.91$ & 0.011 \\
\hline \multicolumn{4}{|l|}{ Cardiac function } \\
\hline NYHA class II & $11(24.44 \%)$ & $17(53.13 \%)$ & \multirow{2}{*}{0.019} \\
\hline NYHA class III & $34(75.56 \%)$ & $15(46.87 \%)$ & \\
\hline \multicolumn{4}{|l|}{ Medications } \\
\hline ACEI (\%) & $8(19.03 \%)$ & $3(9.38 \%)$ & 0.345 \\
\hline$\beta$-blockers (\%) & $18(40.00 \%)$ & $4(12.50 \%)$ & 0.011 \\
\hline Digoxin (\%) & $25(55.56 \%)$ & 0 & $<0.001$ \\
\hline Diuretics (\%) & $45(100 \%)$ & $18(58.25 \%)$ & $<0.001$ \\
\hline
\end{tabular}

ACEI, angiotensin-converting enzyme inhibitors; AF, atrial fibrillation; AR, aortic regurgitation; AS, aortic stenosis; BP, blood pressure; bpm, beats per minute; MR, mitral regurgitation; MS, mitral stenosis; NYHA, New York Heart Association; SR, sinus rhythm.

TABLE 3: Laboratory assessments of patients.

\begin{tabular}{|c|c|c|c|}
\hline & $\mathrm{AF}(n=45)$ & $\mathrm{SR}(n=32)$ & $P$ \\
\hline \multicolumn{4}{|l|}{ Blood measurements } \\
\hline $\mathrm{Hb}(\mathrm{g} / \mathrm{L})$ & $129.17 \pm 11.84$ & $131.61 \pm 8.70$ & 0.353 \\
\hline $\operatorname{ALT}(\mathrm{U} / \mathrm{L})$ & $27.24 \pm 15.22$ & $22.86 \pm 8.41$ & 0.127 \\
\hline $\mathrm{Cr}(\mu \mathrm{mol} / \mathrm{L})$ & $87.37 \pm 13.51$ & $82.04 \pm 19.64$ & 0.182 \\
\hline $\mathrm{LDL}-\mathrm{C}(\mathrm{mmol} / \mathrm{L})$ & $2.47 \pm 0.69$ & $2.45 \pm 0.57$ & 0.887 \\
\hline FBG (mmol/L) & $8.81 \pm 1.28$ & $8.52 \pm 0.84$ & 0.257 \\
\hline INR & $1.44 \pm 0.73$ & $0.99 \pm 0.10$ & $<0.001$ \\
\hline $\mathrm{BNP}(\mathrm{pg} / \mathrm{mL})$ & $139.07 \pm 94.06$ & $61.86 \pm 39.15$ & $<0.001$ \\
\hline Ang I (ng/mL) & $2.13 \pm 0.73$ & $1.65 \pm 0.54$ & 0.003 \\
\hline Ang II (pg/mL) & $328.45 \pm 145.16$ & $217.46 \pm 138.96$ & 0.002 \\
\hline Aldosterone (ng/mL) & $0.17 \pm 0.03$ & $0.17 \pm 0.06$ & 0.840 \\
\hline \multicolumn{4}{|l|}{ Echocardiography } \\
\hline $\mathrm{LAD}(\mathrm{cm})$ & $5.61 \pm 0.88$ & $4.48 \pm 1.21$ & $<0.001$ \\
\hline $\mathrm{RAD}(\mathrm{cm})$ & $4.88 \pm 0.46$ & $4.47 \pm 0.64$ & 0.006 \\
\hline $\operatorname{LVD}(\mathrm{cm})$ & $5.15 \pm 0.82$ & $5.17 \pm 0.91$ & 0.930 \\
\hline $\operatorname{RVD}(\mathrm{cm})$ & $2.35 \pm 0.46$ & $2.32 \pm 0.60$ & 0.863 \\
\hline LVPW $(\mathrm{cm})$ & $0.85 \pm 0.16$ & $0.81 \pm 0.20$ & 0.382 \\
\hline $\operatorname{IVS}(\mathrm{cm})$ & $0.85 \pm 0.16$ & $0.84 \pm 0.19$ & 0.840 \\
\hline $\operatorname{LVEF}(\%)$ & $51.08 \pm 4.62$ & $58.86 \pm 4.16$ & $<0.001$ \\
\hline
\end{tabular}

AF, atrial fibrillation; ALT, alanine transferase; Ang, angiotensin; BNP, brain natriuretic peptide; Cr, creatinine; FBG, fast blood glucose; Hb, hemoglobin; INR, international normalized ratio; IVS, interventricular septum; LDL-C, low-density lipoprotein cholesterol; LAD, left atrial dimension; LVD, left ventricular dimension; LVEF, left ventricular ejection fraction; LVPW, left ventricular posterior wall; RAD, right atrial dimension; RVD, right ventricular dimension; SR, sinus rhythm. 


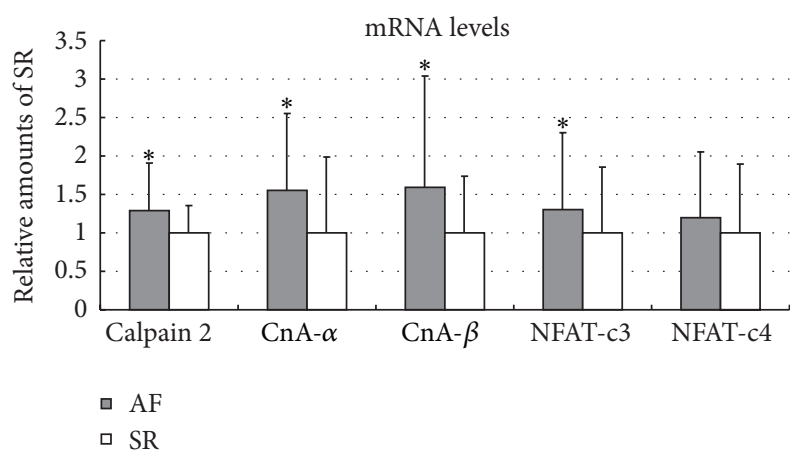

Figure 1: The mRNA expressions of calpain 2, CaN, and NFATs in left atrial tissues isolated from patients with AF and SR. In comparison with SR subjects, significantly increased mRNA levels of calpain $2(128.37 \pm 63.01 \%$ versus $100 \pm 36.07 \%, P=0.015)$, $\mathrm{CnA} \alpha(154.90 \pm 100.38 \%$ versus $100 \pm 98.44 \%, P=0.020), \mathrm{CnA}$ $\beta(159.16 \pm 144.34 \%$ versus $100 \pm 73.36 \%, P=0.037)$, and NFAT-c3 $(130.79 \pm 100.00 \%$ versus $100 \pm 85.48 \%, P=0.028)$, but not NFAT-c4 $(119.40 \pm 85.51 \%$ versus $100 \pm 89.82 \%, P=0.340)$, were observed in patients with $\mathrm{AF} .{ }^{*} P<0.05$ versus $\mathrm{SR}$. AF indicates atrial fibrillation; $\mathrm{CnA}$ alpha and $\mathrm{CnA}$ beta indicate $\alpha$ and $\beta$ isoforms of catalytic $\mathrm{A}$ subunit in calcineurin; NFAT indicates nuclear factor of activated T cells; SR indicates sinus rhythm.

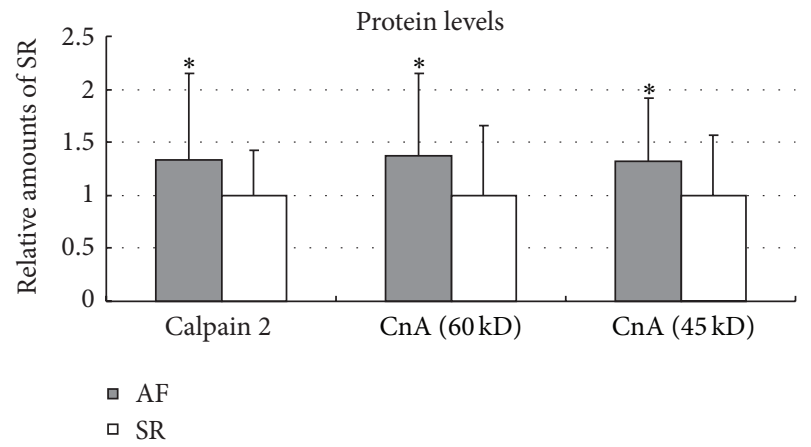

FIGURE 2: The protein expressions of calpain 2, full-length CnA $(60 \mathrm{kD})$, and truncated $\mathrm{CnA}(45 \mathrm{kD})$ in left atriums of patients with $\mathrm{AF}$ and SR. In the protein levels, statistical elevation of calpain 2 (133.68 $\pm 80.82 \%$ versus $100 \pm 42.12 \%, P=0.020)$, full-length CnA $(60 \mathrm{kD})(137.47 \pm 77.61 \%$ versus $100 \pm 65.17 \%, P=0.029)$, and truncated CnA without autoinhibitory domain $(45 \mathrm{kD})(132.40 \pm$ $59.94 \%$ versus $100 \pm 56.19 \%, P=0.019$ ) were found in patients with AF in comparison with SR subjects. ${ }^{*} P<0.05$ versus SR. $\mathrm{AF}$ indicates atrial fibrillation; $\mathrm{CnA}$ indicates catalytic A subunit of calcineurin; SR indicates sinus rhythm.

than NFAT-c4 might contribute toward the development of AF.

3.3. The Protein Expressions of Calpain 2 and CaN. As demonstrated in Figure 2, the protein levels of calpain 2 in atrial samples from patients with AF were higher than those with SR $(133.68 \pm 80.82 \%$ versus $100 \pm 42.12 \%, P=$ $0.020)$. In addition, the protein expression increased in both full-length $\mathrm{CnA}(60 \mathrm{kD})$ and its truncated fragment without autoinhibitory domain $(45 \mathrm{kD})$ in patients with AF $(137.47 \pm$ $77.61 \%$ versus $100 \pm 65.17 \%, P=0.029 ; 132.40 \pm 59.94 \%$ versus $100 \pm 56.19 \%, P=0.019$, resp.). Such results further indicated that calpain 2-CaN pathway might regulate the development of AF through its transcriptional effects.

3.4. Localization of Calpain 2 in Left Atrium. Although cardiomyocyte is most dominant in the heart, other cell types exit, such as smooth muscle cells, endothelial cells, and fibroblasts. Thus, in our current study, we investigated the localization of calpain 2 by immunohistochemistry staining and defined whether it is involved in the development of AF in cardiomyocytes. As shown in Figure 3, brown granules indicating calpain 2 were only visualized in the cytoplasm of atrial myocytes, but not in endocardium or epicardium. An increase in calpain 2 staining was also observed in cardiac samples from patients with AF compared to those from SR group (Figure 3).

\section{Discussion}

Atrial fibrillation (AF) is a common severe arrhythmia and often develops in patients with VHD and diabetes. Permanent $\mathrm{AF}$ is associated with decreased cardiac function and is an independent predictor of heart failure [9]. Calpains have been proposed to be actively involved in the occurrence of AF; however, the molecular mechanisms are not well clarified in human beings. Thus, our current study investigated the role of calpain 2-CaN-NFAT pathway in mediating the development of AF in the patients with VHD and diabetes. Our results indicated that the gene expressions of calpain 2, CaN, and NFAT-c3 but not NFAT-c4 were significantly increased in the left atrial tissue of AF patients with VHD and diabetes compared to those with SR. The upregulated gene expressions of calpain 2 and $\mathrm{CaN}$ responded to an augmented protein levels, and the increased calpain 2 was localized in atrial cardiomyocytes from AF samples, which suggested a positive association of cardiac calpain-CaN-NFAT signaling with AF.

Calpains 1 (or calpain $\mu$ ) and 2 (or calpain $\mathrm{m}$ ) are the two major isoforms of calpain and ubiquitously expressed in the heart. However, calpain 1 was usually activated with a micromolar level of $\mathrm{Ca}^{2+}$, while the activation of calpain 2 requires a higher or pathological millimolar level of intracellular $\mathrm{Ca}^{2+}[10,11]$. Goette et al. previously reported that the level of calpain 1 was increased more than 3 times and the calpain enzymatic activity was doubled in the atrial tissue samples from patients with AF compared to those in SR group [12]. However, the mRNA expressions of calpains 1 and 2 and calpain 2 protein levels between AF and SR patients are identical. A following study further proved the effects of calpain 1 in AF [13]. They found that upregulation of calpain 1, rather than calpain 2, contributes to the activation of calpain. However, our current study indicated that AF is associated with upregulated gene and protein expressions of calpain 2 in the heart tissue. The different results from our current study and other previous reports are probably associated with different cardiac functions and plasma Ang II levels in recruited patients. In our recruited patients, NYHA class III is more prevalent and the patients with AF have a lower LVEF compared to those with SR. Indeed, calpain 2 was significantly increased in NYHA class III or IV failing hearts, but not in class II or nonfailing ones [14]. 


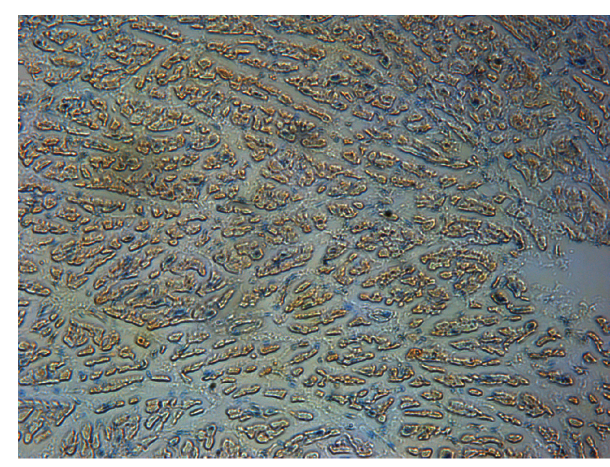

(a)

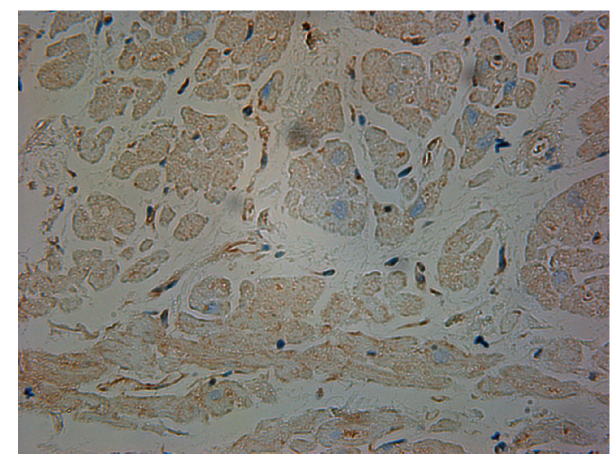

(b)

FIGURE 3: Immunohistochemical localization of calpain 2 in atrial cardiomyocytes ((a) AF group, $\times 200$; (b) SR group, $\times 400)$. Calpain 2 , brown-stained granules, was localized in the cytoplasm of atrial myocytes, but not in endocardium or epicardium. Enhanced expression of calpain 2 was observed in samples from patients with AF compared to those from SR group. AF indicates atrial fibrillation; SR indicates sinus rhythm.

Furthermore, our studies showed that the levels of Ang I and Ang II were significantly elevated in AF group. A previous finding suggested that Ang II regulates calpains in ventricular remodeling [14].

Calpains 1 and 2 can cause limited proteolysis of fulllength $\mathrm{CaN}$ in a $\mathrm{Ca}^{2+}$-dependent manner without the presence of calmodulin [4]. AF induces intracellular calcium overload in atrial cardiomyocytes [15] which may facilitate the activation of CaN-NFAT pathway. The right atrial tissues isolated from VHD patients with AF had upregulated gene and protein expressions in $\mathrm{CaN}$ catalytic and regulatory subunits which contribute to increased activity of the catalytic subunit [16]. NFAT-c3 and NFAT-c4 are the downstream effectors of $\mathrm{CaN}$, which accumulate in the nuclei during $\mathrm{AF}$ due to the increased enzymatic activity of CaN [6]. CnA phosphatase activity and $\mathrm{CnA} \beta$-isoform protein contents were enhanced in patients with chronic AF [17], and the nuclear accumulation of NFAT-c3 protein and the mRNA expression of NFAT-c3 were also upregulated [17]. Our present study demonstrated that the gene and protein expressions of CnA and NFAT-c3 were dramatically elevated in AF patients with VHD and diabetes compared to those with SR. However, it is unclear whether $\mathrm{CnB}$ is also upregulated in $\mathrm{AF}$, though $\mathrm{CnA}$ and $\mathrm{CnB}$ are coexpressed and mutually stabilize each other [18].

It should be noted that pharmacological therapy prior to cardiac surgery might affect calpain pathway in our study. Currently, we do not know whether $\beta$-blockers or diuretics regulate calpain expression; however, digoxin and calcium channel blockers have been tested not affecting calpain 1 expression [12]. Moreover, our recruited patients did not include those with cardiac function NYHA class I or IV. Our future study will expand the patient cohort and identify the role of calpain-CaN-NFAT signaling in mediating AF in all the NYHA classes.

\section{Competing Interests}

The authors declare that they have no competing interests.

\section{Acknowledgments}

This study was supported by grants from the Shandong Provincial Science \& Technology Development Plan Program (Grant no. 2013G0021819), Shandong Provincial Natural Science Foundation (Grant no. ZR2011HM072), Shandong Provincial Natural Science Foundation (Grant no. ZR2010HQ048), Shandong Provincial Medicine and Health Science Technology Development Plan Program (Grant no. 2011BJZD05), Jinan Science \& Technology International Cooperation Project (Grant no. 201401356), and National Natural Science Foundation of China (Grant no. 30900608).

\section{References}

[1] D. D. McManus, M. Rienstra, and E. J. Benjamin, "An update on the prognosis of patients with atrial fibrillation," Circulation, vol. 126, no. 10, pp. e143-e146, 2012.

[2] J. Andrade, P. Khairy, D. Dobrev, and S. Nattel, "The clinical profile and pathophysiology of atrial fibrillation: relationships among clinical features, epidemiology, and mechanisms," Circulation Research, vol. 114, no. 9, pp. 1453-1468, 2014.

[3] A. Bukowska, U. Lendeckel, S. M. Bode-Böger, and A. Goette, "Physiologic and pathophysiologic role of calpain: implications for the occurrence of atrial fibrillation," Cardiovascular Therapeutics, vol. 30, no. 3, pp. e115-e127, 2012.

[4] J.-C. Wang, Y. Zhao, X.-D. Li, N.-N. Zhou, H. Sun, and Y.-Y. Sun, "Proteolysis by endogenous calpain I leads to the activation of calcineurin in human heart," Clinical Laboratory, vol. 58, no. 1112, pp. 1145-1152, 2012.

[5] R. E. A. Musson, C. M. Cobbaert, and N. P. M. Smit, "Molecular diagnostics of calcineurin-related pathologies," Clinical Chemistry, vol. 58, no. 3, pp. 511-522, 2012.

[6] C.-C. Lin, J.-L. Lin, C.-S. Lin et al., "Activation of the calcineurin-nuclear factor of activated T-cell signal transduction pathway in atrial fibrillation," Chest, vol. 126, no. 6, pp. 1926-1932, 2004.

[7] S. Kumar, A. W. Teh, C. Medi, P. M. Kistler, J. B. Morton, and J. M. Kalman, "Atrial remodeling in varying clinical substrates within beating human hearts: relevance to atrial fibrillation," 
Progress in Biophysics and Molecular Biology, vol. 110, no. 2-3, pp. 278-294, 2012.

[8] R. R. Huxley, A. Alonso, F. L. Lopez et al., "Type 2 diabetes, glucose homeostasis and incident atrial fibrillation: the Atherosclerosis Risk in Communities study," Heart, vol. 98, no. 2, pp. 133-138, 2012.

[9] S. Taillandier, A. B. Bernard, B. Lallemand et al., "Prognosis in patients hospitalized with permanent and nonpermanent atrial fibrillation in heart failure," American Journal of Cardiology, vol. 113, no. 7, pp. 1189-1195, 2014.

[10] E. Letavernier, L. Zafrani, J. Perez, B. Letavernier, J.-P. Haymann, and L. Baud, "The role of calpains in myocardial remodelling and heart failure," Cardiovascular Research, vol. 96, no. 1, pp. 38-45, 2012.

[11] C. Patterson, A. L. Portbury, J. C. Schisler, and M. S. Willis, "Tear me down: role of calpain in the development of cardiac ventricular hypertrophy," Circulation Research, vol. 109, no. 4, pp. 453-462, 2011.

[12] A. Goette, M. Arndt, C. Röcken et al., "Calpains and cytokines in fibrillating human atria," American Journal of PhysiologyHeart and Circulatory Physiology, vol. 283, no. 1, pp. H264H272, 2002.

[13] B. J. J. M. Brundel, J. Ausma, I. C. van Gelder et al., "Activation of proteolysis by calpains and structural changes in human paroxysmal and persistent atrial fibrillation," Cardiovascular Research, vol. 54, no. 2, pp. 380-389, 2002.

[14] D. Yang, S. Ma, Y. Tan et al., "Increased expression of calpain and elevated activity of calcineurin in the myocardium of patients with congestive heart failure," International Journal of Molecular Medicine, vol. 26, no. 1, pp. 159-164, 2010.

[15] M. Greiser and U. Schotten, "Dynamic remodeling of intracellular $\mathrm{Ca}^{2+}$ signaling during atrial fibrillation," Journal of Molecular and Cellular Cardiology, vol. 58, no. 1, pp. 134-142, 2013.

[16] F. Zhao, S. Zhang, L. Chen et al., "Calcium- and integrinbinding protein-1 and calcineurin are upregulated in the right atrial myocardium of patients with atrial fibrillation," Europace, vol. 14, no. 12, pp. 1726-1733, 2012.

[17] A. Bukowska, U. Lendeckel, D. Hirte et al., "Activation of the calcineurin signaling pathway induces atrial hypertrophy during atrial fibrillation," Cellular and Molecular Life Sciences, vol. 63, no. 3, pp. 333-342, 2006.

[18] S. A. Parsons, D. P. Milla, B. J. Wilkins et al., "Genetic loss of calcineurin blocks mechanical overload-induced skeletal muscle fiber type switching but not hypertrophy," The Journal of Biological Chemistry, vol. 279, no. 25, pp. 26192-26200, 2004. 


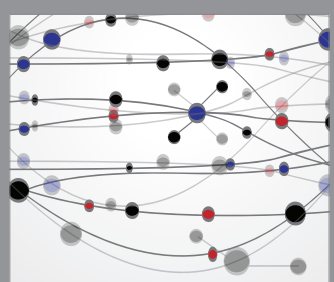

The Scientific World Journal
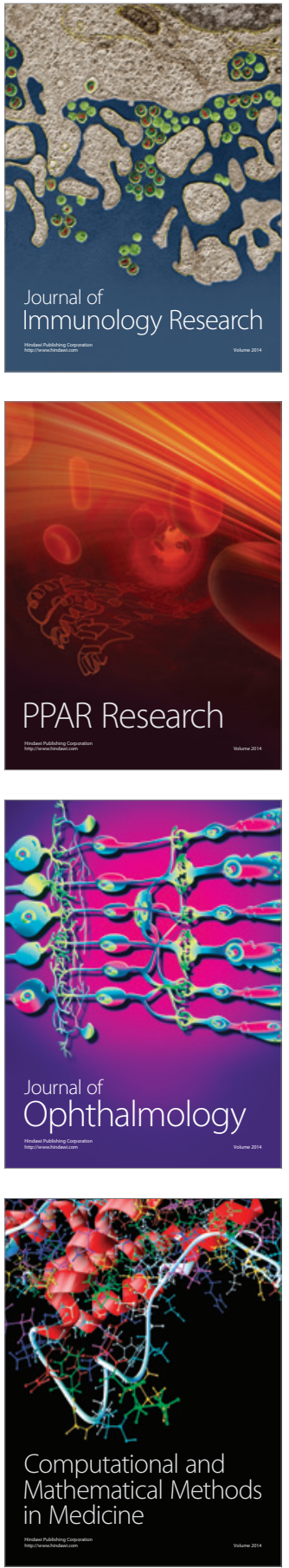

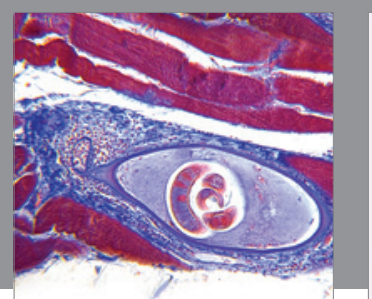

Gastroenterology Research and Practice

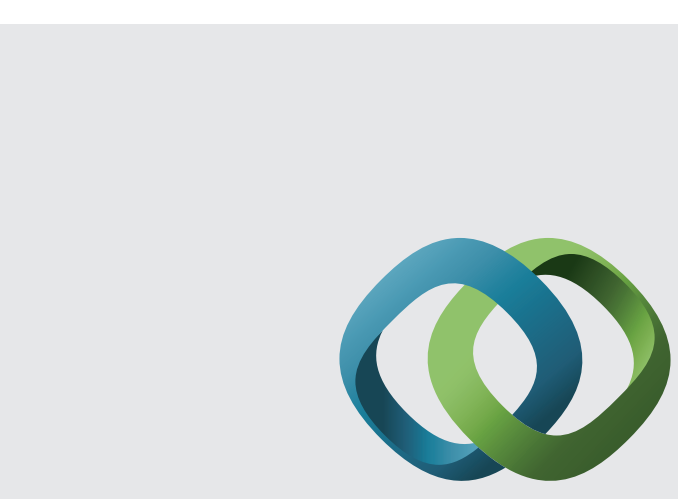

\section{Hindawi}

Submit your manuscripts at

http://www.hindawi.com
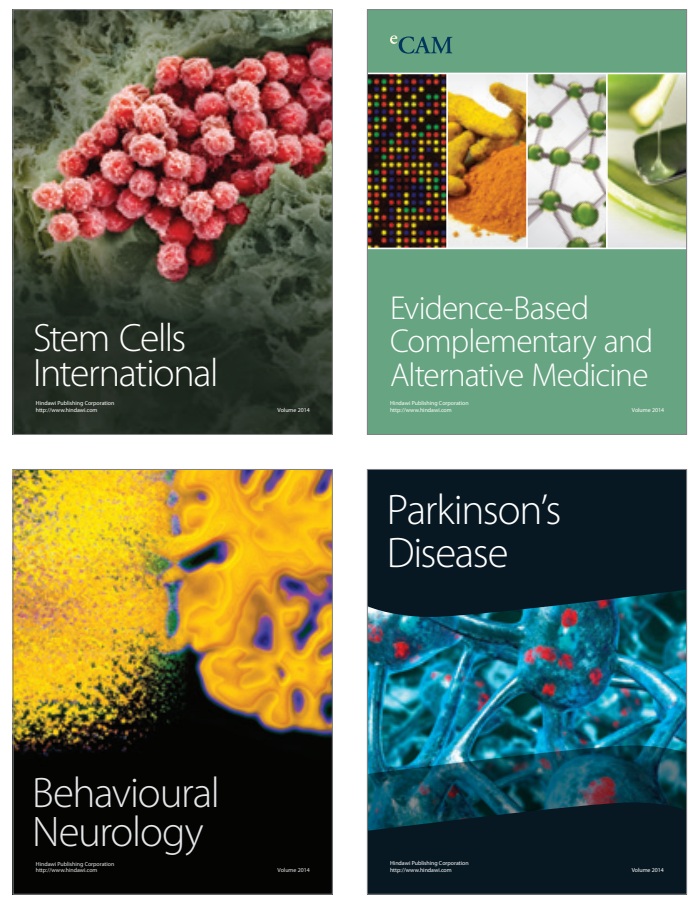
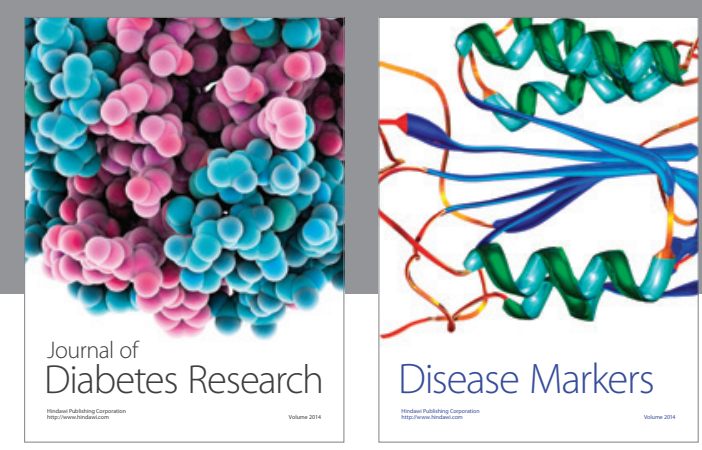

Disease Markers
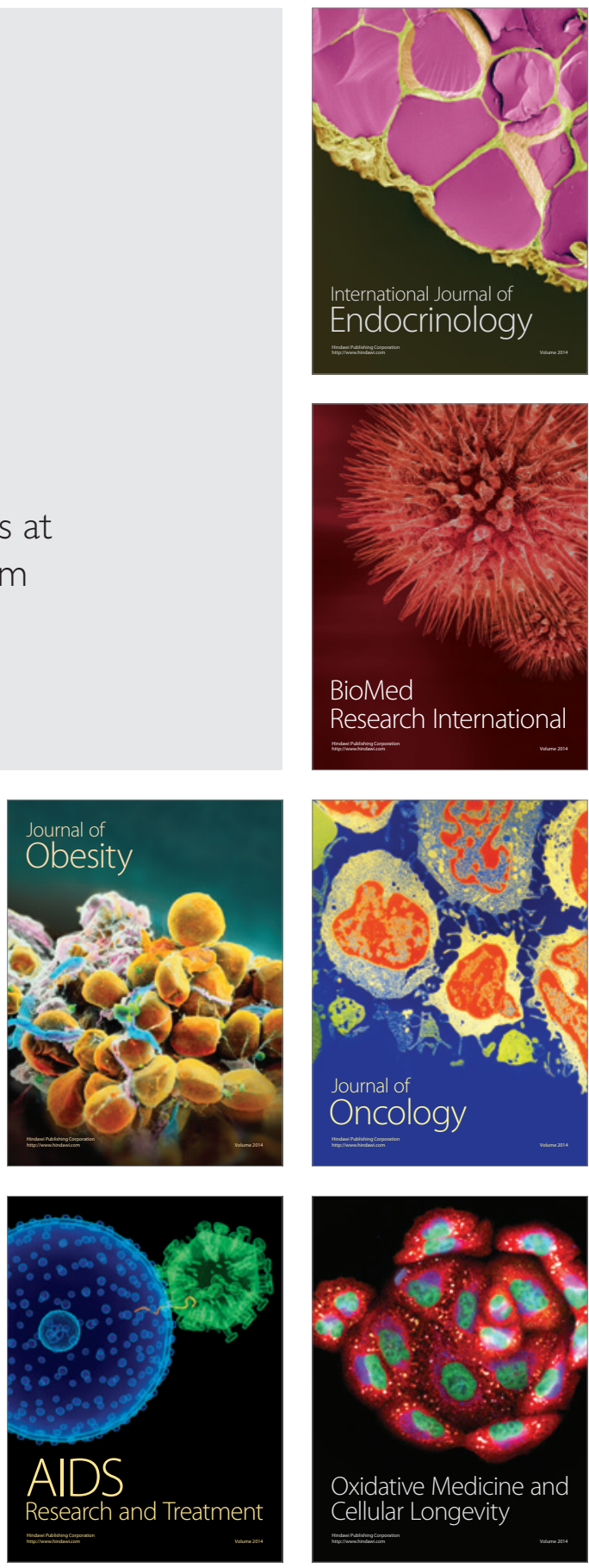